\title{
Meshing for Exhaust System Design on L12B Engine
}

\author{
${ }^{1}$ Fitria Hidayanti, ${ }^{2}$ Erna Kusuma Wati, ${ }^{3}$ Kreshna Agusta Mulyo Adi \\ Engineering Physics Department, Universitas Nasional, Jakarta 12520 Indonesia \\ fitriahidayanti@gmail.com ${ }^{1}$, ernakusuma.w@gmail.com ${ }^{2}$, mulyoadi97@ gmail.com3
}

\begin{abstract}
The CFD simulation test carried out on the meshing for prototype exhaust. To understand the importance of this in studying the making of racing exhaust design, all analyses conducted on 12 types of 3D racing exhausts were made to have a relatively good or decent mesh quality, this is evidenced by the metric mesh value attached to the attachment of 1 mesh parameter.
\end{abstract}

Key words: Meshing, Exhaust, System, Design, Engine

\section{INTRODUCTION}

Discussing the grid generation then discusses one of the most important things in conducting CFD simulation-based research, namely meshing. Meshing is the process of retraining or discrete in a continuous fluid domain to be converted into a discrete computing domain. Meshing is the main requirement determining the success of CFD simulation [1-5].

The size and smallness of its process will determine the accuracy of the calculation result. The easier logic is that the smaller the rationing process will be the more accurate the calculation result, but if too much ization it will give too much calculation load, so the time for calculation/computing takes a long time.

Broadly that is the process of working a meshing, where if you want to analyze a phenomenon related to fluids with CFD software, then it is necessary to pay attention to the meshing process, because of the meshing process determine the accuracy of CFD analysis results in describing the fluid phenomena analyzed, but a large number of meshing elements also needs to be considered given a large amount of meshing will affect computing time, too long computing time will lead to an increase in the cost of electricity usage load [6-11].

Through the previous explanation, in meshing the level of accuracy needs to be taken into account by adding the number of meshing elements, but the number of meshing elements must remain controlled in number as it will impact the length of computing time. So with the problem of accuracy and computational time, a benchmark can be a benchmark to determine whether the meshing is optimal in quantity and quality to provide comprehensive calculation results with good visualization of simulation results along with optimal computing time.

However, before discussing benchmarks that are used as a benchmark for quality as mesh, it is necessary to understand in advance that two types of mesh are popular and often used to solve simulation-based engineering cases [12-19].

\section{MATERIALS AND METHODS}

In this research, the initial hypothesis has been established, the research steps for racing exhaust design begin with measuring the width of the exhaust head port dimensions on the L12B engine block, this is done so that the dimensions of the exhaust head port can be used as a reference in the $3 \mathrm{D}$ process of modelling the exhaust downpipe or exhaust downpipe using CAD software, this is done so that the downpipe is made to match the exhaust size head port on the L12B engine so that the downpipe can be attached to the exhaust head port of the L12B engine.

\section{RESULTS AND DISCUSSION}

Two types of mesh that are popular and often used to solve simulation-based engineering cases, namely:

1. Hexahedron. The hexahedron form is a favourite mesh form of CFD software operators, its shape considered ideal and orderly in shaping each computing domain if achieved then mesh quality and computing time become efficient accompanied by the results of visualization of fluid simulations with very high resolution.
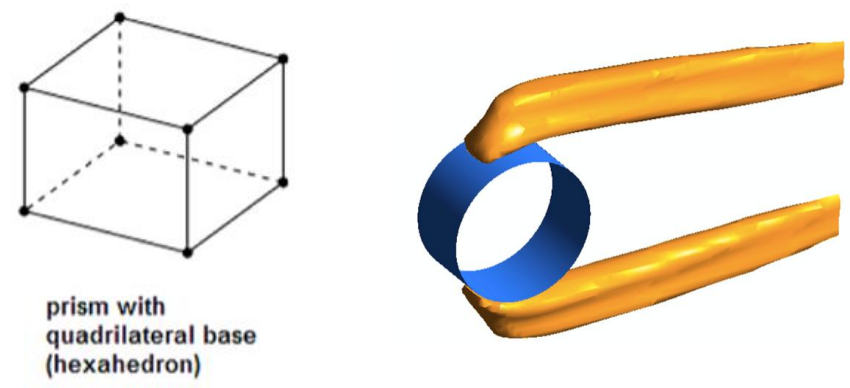

Figure 1: Hexahedron mesh shapes and fluid visualizations with hexahedron mesh 
However, the ideal shape of a mesh can only be applied to simple geometric shapes, in other words hexahedron mesh shapes have difficulty following field shapes or objects that have geometry with extreme curves, whereas almost all objects in the engineering world have diverse geometric shapes with unique extreme curves.
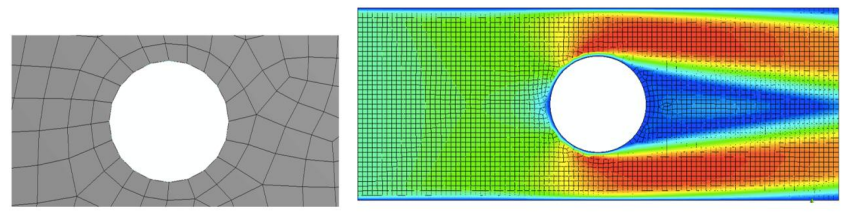

Figure 2: An example of applying to mesh using a hexahedron mesh shape.

2. Tetrahedron. Based on the reference to the lack of hexahedron mesh form, a more adaptive mesh form was developed, namely the tetrahedron form. Although it has a more adaptive ability to geometric shapes with extreme curves, tetrahedron mesh shapes require greater computer memory usage than hexahedron shapes. This is due to the lack of tetrahedron form in filling a volume of the same element size, with tetrahedron mesh required a number of elements that are 2 or 3 times more than hexahedron, in addition, the tetrahedron mesh shape takes longer computing time than the hexahedron mesh shape since the tetrahedron mesh shape has only 3 main nodal (triangular shape) and the hexahedron mesh shape has 4 main nodes (square shape).

The number of nodal is very influential in the speed of convergence, and the more nodal, the faster a compute leads to convergent results so it is logical that the tetrahedron mesh form takes longer computing time than the hexahedron mesh form.

In addition to requiring longer computational time, the visualization of fluid flow simulation produced by tetrahedron mesh has a lower resolution than the visualization results computed using hexahedron mesh. However, the mesh tetrahedron form is considered to provide more accurate results than hexahedrons if applied to objects that have a "strange" and extreme shape.

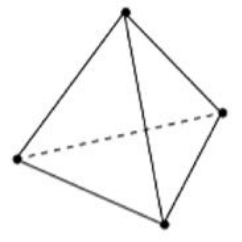

tetrahedron

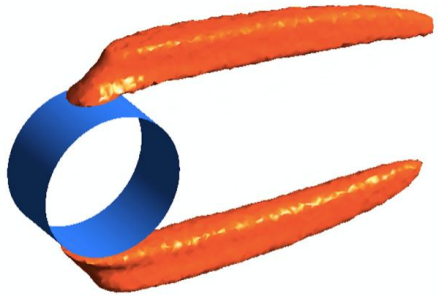

Figure 3: Tetrahedron mesh shape and fluid visualization using tetrahedron mesh
Based on images 29 and 31, it can be seen that the difference in mesh shape used will greatly affect the results of CFD software simulation visualization, so it can be concluded that the hexahedron mesh shape is used to produce simulation visualization results because it produces high resolution, while the tetrahedron mesh form is used to produce more comprehensive fluid mechanics simulation computational results with a lower error rate when applied to objects that have complex geometry.

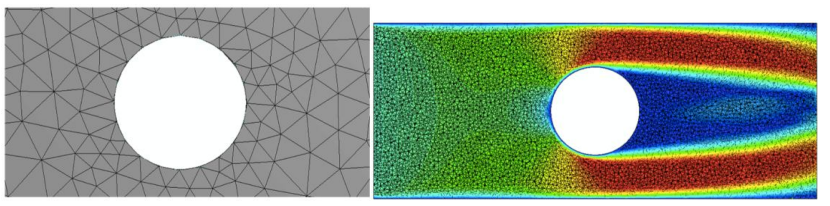

Figure 4: Applying to mesh using a tetrahedron mesh shape

When looking at images 30 and 32, it can be seen that by the naked eye the number of elements of the hexahedron mesh has a fewer amount than the tetrahedron mesh, as a result of this in addition to causing differences in computing time, the use of RAM memory on the computer will also be affected, so that the speed or slow of the computing process is also slightly determined by the specifications of the computer used. After knowing the type of mesh, the next step is to know the quality benchmark of a mesh by paying attention to 4 benchmark meshing parameters.

The 4 benchmark parameters consist of:

1. Aspect Ratio. The size of the cell stretch, calculated as the ration between the maximum and minimum values of the distance between the centroid cell, the face centroid and the distance between the centroid cell and the node. The benchmark aspect ratio value that produces high-quality mesh if the benchmark value is below or equal to 5 .

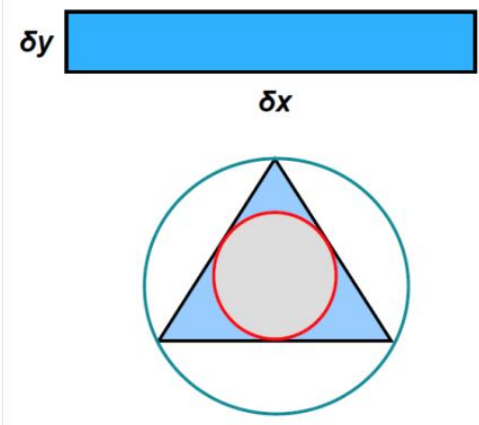

Figure 5: The 2-dimensional shape of the circle is meshed with the type of tetrahedron mesh, from the tetrahedron mesh then the red circle is the part calculated numerically by the CFD software, along with the aspect ratio picture.

2. Orthogonal Quality. The concept of this relates to a close angle between the element faces and the relevant optimal angle, an orthogonal quality benchmark value that produces a high quality mesh if the benchmark value is close to or equal to 1 . 
Fitria Hidayanti et al., International Journal of Emerging Trends in Engineering Research, 9(6), June 2021, 728 - 732

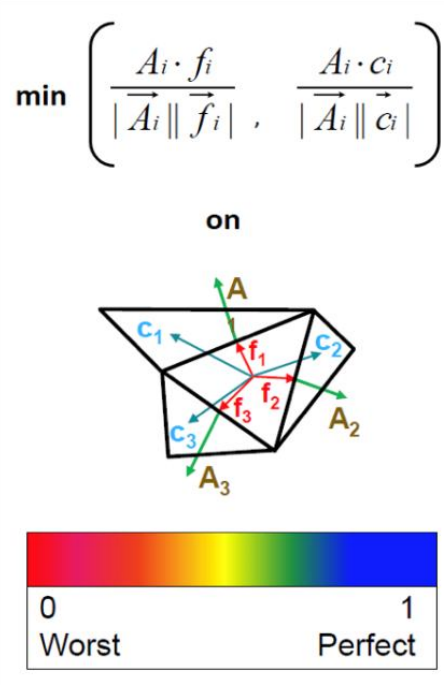

Figure 6: Orthogonal quality metrics

3. Skewness. It is defined as the difference in the shape of each cell that exists at the time of the meshing process against symmetrical cell shapes on the same volume object. The process of calculating skewness values is done by comparing between the corners of meshing cell elements that are formed and compared to normal angles. By obtaining a minimum angle value between the two overlapping lines on the formed meshing element, the two lines consist of a reference line that has a reference angle and a mesh line that has a mesh angle (reference angle 90 for hexahedron mesh type, 60 for tetrahedron mesh type). If the reference angle is reduced by the mesh angle, it gets the skewness value. A benchmark value that produces a high-quality mesh if the benchmark value is close to or equal to 0 .

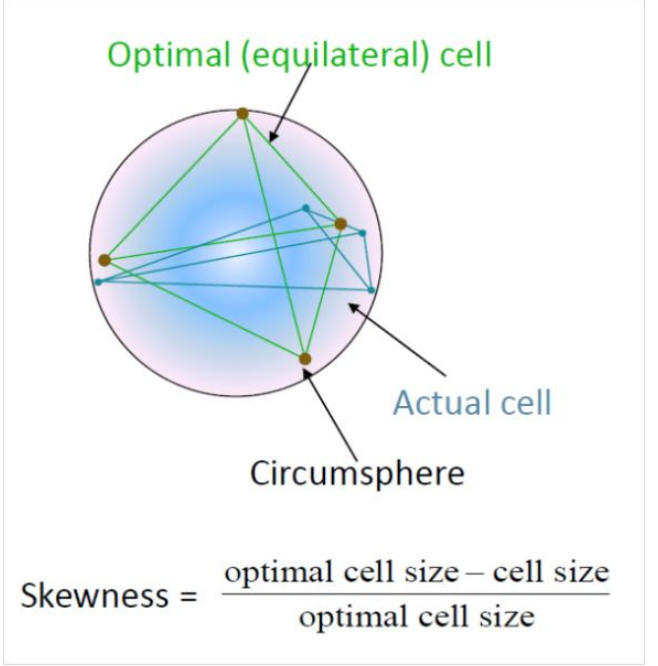

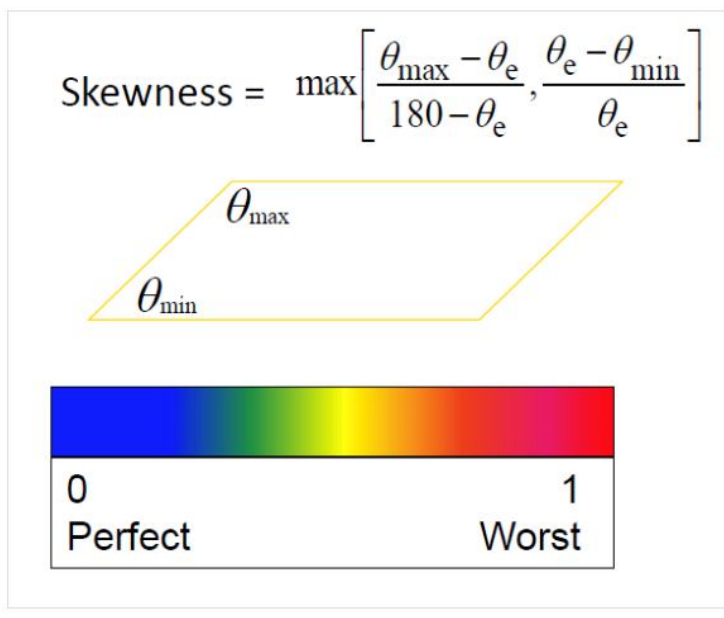

Figure 7: Skewness metrics

4. Element Quality. The combination of aspect ratio, orthogonal quality and skewness produce mesh elements that can be measured in quality or have other element quality names. This parameter is strongly influenced by the quality of the previous 3 parameters, if the quality of the previous parameters is good enough, then it produces a mesh called structured mesh or structured mesh.

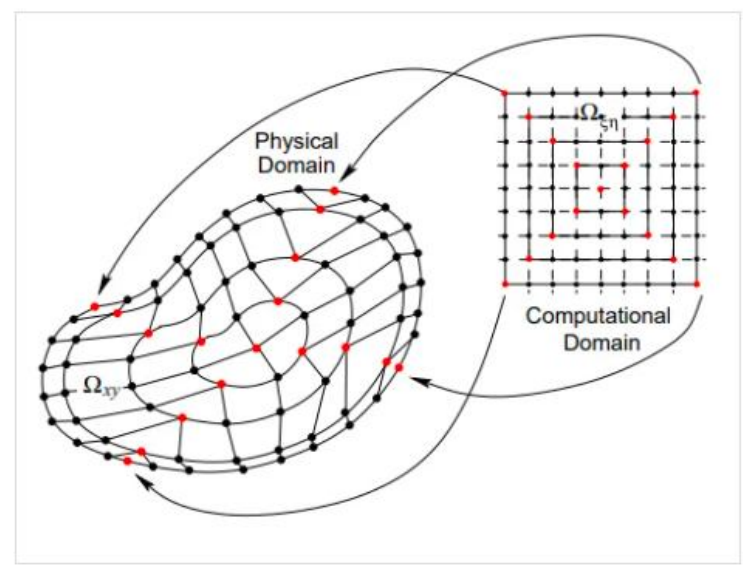

Figure 8: Structured mesh

A structured mesh is a structure-formed mesh in which each nodal will be arranged in a patterned way to form a computational domain structure. With the formation of computational domains structured, Navier-stokes equation computational computation can be solved faster with a high degree of accuracy because the Navier-Stokes equation can be calculated up to the top of order 1, which will produce computing results that have very high accuracy, the structured mesh also has other capabilities that can perform time-dependent / transient computing where this condition is close to the conditions that occur in the real world, where the whole case in the engineering world must be a time-dependent case.

The opposite of a structured mesh, unstructured mesh or unstructured mesh is a mesh that is unable to form a 
computational domain, so this type of mesh is very simple. Because of its simplicity, this type of mesh has the advantage that it is easy to create or generate by anyone, even by people who do not yet have the basic science of meshing and CFDs, another advantage of unstructured mesh is that each computing node is composed following the original form of the physical model, so that computed calculations according to the point at which the nodal is located, therefore the visualization of computational results can result in higher resolution and animation quality than structured mesh.

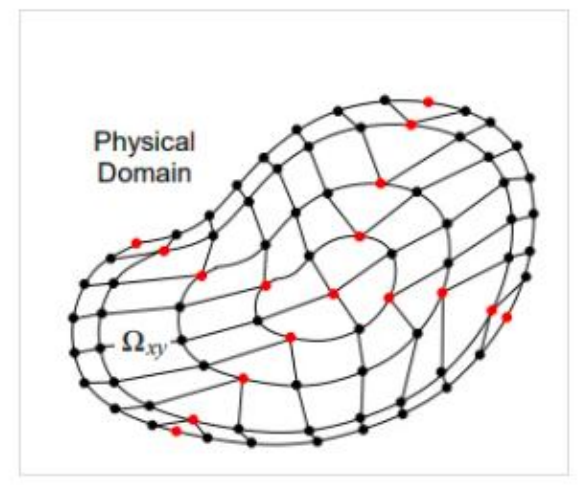

Figure 9: Unstructured mesh

However, the flaw of the first and foremost unstructured mesh is that it has an $80 \%$ computational error resulting in computational errors so that in other words the computational results are inaccurate, the second flaw of unstructured mesh is that almost the entire computational calculation of the Navier-Stokes equation can only be computed in order 1, unable to achieve a higher-order so that this will affect the accuracy of computational results. Lastly, the disadvantage of unstructured mesh is the opposite of structured mesh, i.e. unstructured mesh which has computational error unrest of $80 \%$ causing this type of mesh to be unable to perform time-dependent or transient based computing, so almost all computing using unstructured mesh based on steady-state or deaf condition, this is done because the computing error can be minimized if done in a blind condition.

\section{CONCLUSION}

Based on the difference between structured mesh and unstructured mesh depicted by images 3.37 and 3.38, it can be concluded that a mesh that has a good element of quality is a structured mesh with a value close to or equal to 1 . If these four parameters meet the specified benchmark value, then it can be ascertained that computational results with CFD software produce research results that are $80 \%$ to $95 \%$ accurate close to the experimental test results, with this achieving, then the cost of experimental trials can be reduced with CFDs.

\section{ACKNOWLEDGEMENT}

Thank you for Lembaga Penelitian dan Pengabdian kepada Masyarakat (LPPM) Universitas Nasional and my colleagues at Faculty of Engineering and Science, Universitas Nasional, Jakarta, Indonesia.

\section{REFERENCES}

1. Hidayanti F., Adi, K.A.M., and Wati E.K. Developing Racing Exhaust System Performance using Computational Fluid Dynamics Software. AIP Conference Proceedings. American Institute of Physics. The United States. 2262. 003016. 2020.

2. Abdessemed, C., Bouferrouk, A., \& Yao, Y. Aerodynamic and Aeroacoustic Analysis of a Harmonically Morphing Airfoil Using Dynamic Meshing. In Acoustics (Vol. 3, No. 1, pp. 177-199). Multidisciplinary Digital Publishing Institute. 2021

3. Pewowaruk, R., Li, Y., Rowinski, D., \& Roldán $\square$ Alzate, A. Solution Adaptive Refinement of Cut $\square$ Cell Cartesian Meshes Can Improve FDA Nozzle Computational Fluid Dynamics Efficiency. International Journal for Numerical Methods in Biomedical Engineering, e3432.

4. Wang, L., Robertson, A., Jonkman, J., Yu, Y. H., Koop, A., Borràs Nadal, A., ... \& Sarlak, H. Investigation of nonlinear difference-frequency wave excitation on a semisubmersible offshore-wind platform with bichromatic-wave CFD simulations. In International Conference on Offshore Mechanics and Arctic Engineering (Vol. 84768, p. V001T01A009). American Society of Mechanical Engineers. 2021.

5. Eppinger, T., \& Wehinger, G. D. A Generalized Contact Modification for Fixed $\square$ Bed Reactor CFD Simulations. Chemie Ingenieur Technik,93(1-2), 143-153. 2021.

6. Warda, H. A., Wahba, E. M., \& El-Din, M. S. Computational Fluid Dynamics (CFD) simulation of liquid column separation in pipe transients. Alexandria Engineering Journal,59(5), 3451-3462. 2020.

7. Ferziger, M. P. Introduction to Numerical Methods. in Computarional Methods for Fluid Dynamics, New York, Springer Publishing Group 21-35. 2002.

8. de Vasconcellos Araújo, M., Raquel de Brito Correia, B., Alves Agra Brandão, V., Rodrigues de Oliveira, I., Santos, R. S., Luiz de Oliveira Neto, G., ... \& Gilson Barbosa de Lima, A. Convective drying of ceramic bricks by CFD: Transport phenomena and process parameters analysis. Energies, 13(8), 2073. 2020. 
9. Heydari, O., Sahraei, E., \& Skalle, P. Investigating the impact of drillpipe's rotation and eccentricity on cuttings transport phenomenon in various horizontal annuluses using computational fluid dynamics (CFD). Journal of Petroleum Science and Engineering, 156, 801-813. 2017.

10. Adaze, E., Al-Sarkhi, A., Badr, H. M., \& Elsaadawy, E. Current status of CFD modeling of liquid loading phenomena in gas wells: a literature review. Journal of Petroleum Exploration and Production Technology, 9(2), 1397-1411. 2019.

11. Jeong, J. H., Song, M. S., \& Lee, K. L. CFD investigation of three-dimensional flow phenomena in a JAEA 127-pin wire-wrapped fuel assembly. Nuclear Engineering and Design, 323, 166-184. 2017.

12. Onebe K. O. Assessment of Effect of Back Pressure and Gas Flow Dynamics of Exhaust Pipe of a Two-Stroke Engine on Its Performance Characteristics. Journal of Emerging Technologies and Innovative Research (JETIR). 3(4). 223-234. 2016.

13. Sitaraman, J., Lakshminarayan, V. K., Roget, B., \& Wissink, A. M. Progress in strand mesh generation and domain connectivity for dual-mesh cfd simulations. In 55th AIAA Aerospace Sciences Meeting (p. 0288). 2017.

14. Munson, O. H. R. Computational Fluid Dynamics. in Fundamentals of Fluid Mechanics (Seventh Edition), New York, John Wiley \& Sons, Inc., 725. 2013.

15. Bao, H., Feng, J., Dinh, N., \& Zhang, H. Deep learning interfacial momentum closures in coarse-mesh CFD two-phase flow simulation using validation data. International Journal of Multiphase Flow, 135, 103489. 2021.

16. Mohammadi, M. A., \& Jafarian, A. CFD simulation to investigate hydrodynamics of oscillating flow in a beta-type Stirling engine. Energy, 153, 287-300. 2018.

17. Saleem, A., Farooq, S., Karimi, I. A., \& Banerjee, R. A CFD simulation study of boiling mechanism and BOG generation in a full-scale LNG storage tank. Computers \& Chemical Engineering, 115, 112-120. 2018.

18. Gu, B., Adjiman, C. S., \& Xu, X. Y. The effect of feed spacer geometry on membrane performance and concentration polarisation based on 3D CFD simulations. Journal of Membrane Science, 527, 78-91. 2017.

19. Shuchitangshu Chatterjee, Sumit Kumar Dubey. 3D Modeling \& Numerical Simulation of Heat transfer of Back-pack Thermoelectric Cooling Helmet validated By Experimentation at Hot
Environment. International Journal of Emerging Trends in Engineering Research, 6(8). 2018. 